\title{
Les Annales françaises de médecine d'urgence, la médecine d'urgence pédiatrique et Toulouse récompensés !
}

\author{
The Annales françaises de médecine d'urgence, paediatric emergency medicine, \\ and Toulouse are rewarded!
}

\author{
G. Chéron - B. Riou \\ (C) SFMU et Springer-Verlag France 2011
}

Nous avons le très grand plaisir de vous annoncer que le Syndicat de la presse et de l'édition des professions de santé (SPEPS) a décerné son premier prix, dans la catégorie « travaux originaux », à un article paru dans le tout premier numéro des Annales françaises de médecine d'urgence (AFMU), en janvier 2011 [1]. Cet article intitulé Préparation des services d'accueil des urgences (SAU) français à la prise en charge des urgences vitales de l'enfant est une étude originale de nos collègues de l'hôpital des enfants du centre hospitalo-universitaire de Toulouse, les Drs Isabelle Claudet et Erick Grouteau (Fig. 1).

Les auteurs ont analysé l'organisation de 100 centres d'urgences accueillant des enfants en 2009. Ils se sont intéressés aux moyens en personnel, aux écarts entre les recommandations des sociétés savantes sur les salles d'accueil des urgences vitales (SAUV) et la réalité de terrain, et à la mise en place des protocoles de prise en charge pédiatriques. Le taux de réponse de cette enquête réalisée via le site Web de la Société française de médecine d'urgence (SFMU) et des relances postales est de $33 \%$. Les résultats donnent une vision précise de la réalité des urgences pédiatriques. Les difficultés démographiques ne sont pas passées sous silence et le travail à venir est considérable. Mais, photographie des organisations en place, ce travail souligne les améliorations immédiates possibles : partage de protocoles entre centres spécialisés et centres polyvalents, mise aux normes des équipements. Il illustre les besoins de formations complémen-

\section{G. Chéron}

Service des urgences pédiatriques,

CHU Necker-Enfants-malades,

Assistance publique-Hôpitaux de Paris (AP-HP),

université Paris-Descartes, Paris, France

\section{B. Riou $(\square)$}

Service d'accueil des urgences, CHU Pitié-Salpêtrière,

AP-HP, université Pierre-et-Marie-Curie-Paris-VI,

47-83, boulevard de l'Hôpital,

F-75651 Paris cedex 13, France

e-mail : bruno.riou@psl.aphp.fr

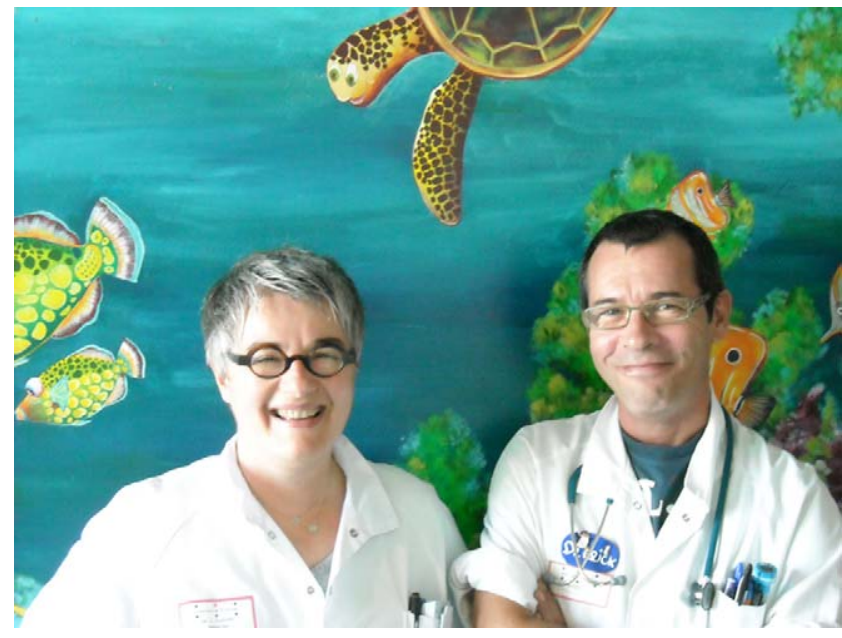

Fig. 1 Les auteurs de l'article Préparation des services d'accueil des urgences (SAU) français à la prise en charge des urgences vitales de l'enfant [1], les Drs Isabelle Claudet et Erick Grouteau $\mathrm{du}$ CHU de Toulouse.

taires à la pédiatrie, et à cet égard, le référentiel métier actuellement présenté dans les instances européennes doit guider les enseignements mis en place. Les urgences pédiatriques sont les urgences de l'enfant, de ses spécificités. L'enfant ne connaît pas les pathologies de l'adulte, et l'adulte ne rencontrera plus nombre des pathologies de l'enfance. Le potentiel de croissance de l'enfant est en soi un des éléments du traitement des pathologies orthopédiques dont les indications diffèrent de celles de l'adulte. Prendre en charge un enfant, c'est en toutes circonstances vouloir préserver ses potentialités et lui permettre de devenir l'adulte autonome qu'il doit devenir. C'est aussi tenir compte des spécificités pharmacologiques propres à cet âge. C'est aussi ce dialogue singulier qui s'établit entre l'enfant, ses parents et le médecin. Néanmoins, il s'agit bien d'une pratique de l'urgence, avec ses difficultés, son savoir-faire, son cadre et nous sommes contents qu'Isabelle Claudet et Erick Grouteau aient soumis ce manuscrit aux AFMU. 
C'est une très grande fierté pour les AFMU de se voir décerner ce prix pour sa première année d'existence et c'est tout simplement aussi une reconnaissance pour la médecine d'urgence, spécialité nouvelle qui démontre ainsi son dynamisme. Le référentiel métier-compétence de la médecine d'urgence qui sera prochainement publié dans les AFMU insiste sur les éléments constitutifs de la médecine d'urgence, dans ses aspects préhospitaliers et hospitaliers, médicaux et chirurgicaux, de médecine de l'adulte et de l'enfant [2]. Il est particulièrement bienvenu que ce prix récompense une de ses composantes essentielles, la médecine d'urgence pédiatrique. Nous souhaitons remercier vivement les auteurs de cet article, Isabelle Claudet et Erick Grouteau, d'abord d'avoir conduit une remarquable étude sur la prise en charge des urgences vitales chez l'enfant dont l'utilité n'a échappé à personne, en tout cas pas au jury de la SPEPS, mais également d'avoir choisi les AFMU pour publier leur manuscrit. Finalement, Isabelle Claudet et Erick Grouteau auront fait un superbe cadeau de naissance aux AFMU, et nous attendons avec impatience les travaux futurs des urgentistes pédiatres, de Toulouse et d'ailleurs, afin de fêter dignement l'anniversaire de cet événement.

\section{Références}

1. Claudet I, Grouteau E (2011) Préparation des services d'accueil des urgences (SAU) français à la prise en charge des urgences vitales de l'enfant. Ann Fr Med Urg 1:5-9

2. Nemitz B, Carli P, Carpentier F, et al (2012) Référentiel métiercompétence de la médecine d'urgence. Ann Fr Med Urg 2 (in press) 